Bol. Acad. peru. leng. 66. 2019 (11-36)

\title{
VIEJOS Y NUEVOS OFICIOS EN LOS DICCIONARIOS DE PERUANISMOS (1938-2015)
}

\section{OLD AND NEW TRADES IN PERUVIANISMS DICTIONARIES (1938-2015)}

\author{
Rosa Luna \\ Universidad Ricardo Palma
}

\section{Resumen:}

El objetivo central de este estudio, de corte empírico, aplicado y documental, fue analizar el comportamiento lexicográfico que presentan las denominaciones de oficios en los diccionarios de peruanismos.

La muestra del estudio estuvo constituida por 525 oficios ubicados en trece obras lexicográficas de peruanismos publicadas entre 1938 y 2015. Para el análisis de los datos, se aplicó la técnica del análisis de contenido. Las tablas de estadística descriptiva se diseñaron con el empleo del software SPSS.

La investigación arribó a las siguientes conclusiones generales: el comportamiento lexicográfico de los oficios en los diccionarios de peruanismos manifiesta una marcada tendencia a la derivación sufijal, con considerable recurrencia de los sufijos -ero/-era. La semitransparencia, la escasa recurrencia en diccionarios y la elevada dispersión temática constituyeron tres características adicionales del corpus lexicográfico de estudio. 
Abstract:

The main objective of this study, of empirical, applied, and documental nature, was to analyze the lexicographic behavior displayed by the names of trades in the dictionaries of Peruavianisms.

The sample of the study was formed by 525 trades extracted from thirteen lexicographic works on Peruavianisms, which were published between 1938 and 2015. The content analysis technique was applied in the data analysis and the descriptive statistics tables were designed with SPSS software.

The study reached the following general conclusions: The lexicographic behavior of the trades in the dictionaries of Peruvianisms show a marked tendency to suffixal derivation with a considerable recurrence of the -ero/-era suffixes. Semitransparency, reduced recurrence in dictionaries, and wide subject dispersion represented three additional characteristics identified in the lexicographic corpora of the study.

Palabras clave: comportamiento lexicográfico; transparencia; sufijación; recurrencia; dispersión temática.

Key words: lexicographic behavior; transparency; suffixal recurrence; subject dispersion.

Fecha de recepción: $\quad$ 10/09/2019

Fecha de aceptación: $\quad 30 / 11 / 2019$

\section{Introducción}

\subsection{Planteamiento del problema}

\subsection{Formulación e importancia}

La preocupación por los oficios en la lexicografía ha sido constante en diferentes lenguas, y el español no ha sido la excepción. Esta 
lengua cuenta con trabajos sobre oficios, profesiones y ocupaciones en sus diferentes variedades dialectales, salvo la peruana. Al tomar conciencia de este escenario, y luego de analizar los oficios incluidos en diversas fuentes lexicográficas peruanas surgió la necesidad de llenar este vacío a partir de la resolución de los siguientes problemas y subproblemas de investigación: ¿Cuál es el comportamiento lexicográfico que presentan los neologismos de oficios incluidos en los diccionarios de peruanismos? Con sus respectivos subproblemas: ¿Qué recursos de formación emplean los oficios recogidos en los diccionarios de peruanismos? ¿Cuál es el sufijo más productivo? ¿Qué grado de transparencia revelan los peruanismos de oficios? ¿A qué campos temáticos pertenecen? ¿Qué diccionario aporta el mayor número de oficios? ¿Qué oficios tiene mayor índice de frecuencia de aparición en las fuentes lexicográficas? ¿Qué oficios presentan mayor alternancia y variación denominativa?

La justificación de esta investigación radica en la necesidad de que los hablantes peruanos, y en especial los traductores, se familiaricen con los oficios característicos del español peruano. Además, el producto de esta pondrá a disposición de los lexicógrafos una base de datos diacrónica del léxico de oficios peruanos (1938-2015), corpus que servirá como insumo para la elaboración de futuros trabajos lexicográficos puntuales sobre esta temática de estudio, y que con el tiempo podrá hacerse extensiva a los oficios privativos de cada una de las 47 lenguas peruanas restantes.

\subsection{Antecedentes}

La mayor parte de antecedentes sobre oficios en fuentes lexicográficas pertenecen a variantes dialectales distintas al español peruano. El trabajo más cercano al tema es el artículo «La cosificación como recurso terminológico de modalidades laborales en la economía peruana»(Luna, 2010) en el que la autora analiza el comportamiento terminológico de catorce oficios informales peruanos, los recursos de formación empleados, entre otros aspectos, y concluye que existe una tendencia a la cosificación en todos ellos. 


\subsection{Marco conceptual y teórico}

Para la construcción del marco teórico, se tomaron en cuenta tres enfoques: los recursos de formación que caracterizan la acuñación del léxico de oficios, el grado de transparencia del léxico y la clasificación de ocupaciones peruanas.

Según Álvarez Esquerra, citado por Muñoz (2010), la sufijación es el procedimiento derivativo más utilizado en español tanto para la formación de palabras como para la creación de neologismos. Este mismo autor agrega que las bases léxicas a las que se adjuntan pueden ser de naturaleza sustantiva, adjetiva o verbal.

Ahora bien, concretamente en lo tocante a la sufijación nominal, son abundantes los autores (Almela, 1999; Cabré, Freixa, y Solé, 2000; Doménech, Estopá, Folia, y Morel 2000; Skolniková, 2008; Rossowová, 2009; Fuentes, Cañete, Gerding, Kotz y Pecchi, 2010) que la consideran como recurso por excelencia para la formación del léxico de ocupaciones. Lacuesta y Bustos, citados por Muñoz (2010), añaden que una particularidad de este tipo de sufijación es que varios sufijos pueden formar nombres de oficios y que -ero destaca como el sufijo más productivo para formar nombres de ocupaciones básicamente rurales o de menor especialización.

Complementariamente, la Nueva gramática de la lengua española (NGLE, 2009) especifica que la relación que se establece entre la base léxica y el sufijo ero/-era es bastante variable. Se trata, indica la gramática, de un sufijo que remite a cosas o a personas que venden, fabrican, preparan, componen, buscan, ofrecen, emplean como herramienta, cuidan, vigilan y cazan.

A juicio de Romero, citado por Muñoz (2010), -ista, a diferencia de -dor/dora y -erolera, se emplea para generar denominaciones de oficios más formales (electricista, ortopedista) y de naturaleza neutra. Siguiendo a Raimer, citado por Muñoz (2010), este último sufijo remite a oficios artísticos, artesanales, 
modernos, musicales, al igual que a acciones, pacientes, productos, instrumentos utilizados, modos, desarrollo y perfeccionamiento de nuevos oficios, mecanización de medios de comunicación e instrumentos, así como a la esfera del ocio. Una característica del sufijo en mención es su naturaleza agentiva, referida exclusivamente a personas. Complementariamente, la NGLE (2009) indica que se emplea como derivado de nombres comunes o propios, y en muy pocos casos como adjetivo.

Siguiendo a la NGLE (2009), el sufijo -dor/dora posee una base verbal, básicamente transitiva, forma sustantivos a partir de verbos, así como adjetivos derivados de sustantivos. Este sufijo hace referencia a nombres de personas, en su mayoría, agentes, instrumentos y lugares.

-Dor/-dora se clasifican en dos categorías, los relativos a episodios, estadios o acciones que se realizan en determinado momento y los caracterizados o de individuo, que se subdividen en a) de hábitos o costumbres y b) de profesiones oficios y ocupaciones. Los oficios a los que aluden pueden ser honorables o no, y un mismo vocablo puede designar a actividades parcial o totalmente distintas.

En lo que respecta a todos los sufijos antes mencionados, cabe señalar que todos ellos experimentan alternancia sufijal, al igual que variantes denominativas y dialectales (NGLE, 2009).

La transparencia léxico-semántica constituye el segundo enfoque seleccionado para el análisis de los resultados. Para Ullmann (1987), en los idiomas, cualquiera que sea su naturaleza, existen palabras tanto transparentes o motivada, fonética, morfológica o semánticamente como opacas o arbitrarias. El autor define a los transparentes como aquellos que dejan ver a través de ellos, y a los opacos como los que no dejan ver a través de ellos. En otras palabras, los transparentes permiten extraer su significado a partir de la descomposición de sus componentes, mientras que los opacos impiden hacerlo de esa manera. 
Finalmente, el tercer enfoque aplicado a este trabajo concierne a la clasificación de ocupaciones peruanas conformada por diez grandes grupos ocupacionales que abarcan desde directores y gerentes hasta ocupaciones militares, pasando por profesionales científicos e intelectuales, técnicos y profesionales de nivel medio, personal de apoyo administrativo, trabajadores de los servicios y vendedores de comercios y mercados, agricultores y trabajadores calificados agropecuarios, forestales y pesqueros, oficiales, operaciones y artes mecánicas y otros oficios, operadores de instalaciones y máquinas y ensambladore, y ocupaciones elementales (INEI, 2015).

\section{Objetivos y variables}

\subsection{Objetivo general}

- Analizar el comportamiento lexicográfico que presentan las denominaciones de oficios en los diccionarios de peruanismos

\subsection{Objetivos específicos}

- Determinar la frecuencia de aparición de oficios en el corpus lexicográfico.

- Identificar los campos temáticos con mayor número de oficios.

- Ubicar los recursos de formación con mayor productividad en la acuñación de oficios.

- Detectar el grado de transparencia de los oficios presentes en el corpus lexicográfico. 


\subsection{Variables, dimensiones e indicadores}

\section{Tabla 1}

Matriz de operacionalización de variables

\begin{tabular}{cc}
\hline VARIABLES & DIMENSIONES \\
\hline ÍNDICE DE FRECUENCIA & Elevada \\
DE APARICIÓN & Promedio \\
Baja & \\
CAMPO TEMÁTICO & Agricultura, ganadería y pesca \\
Albañilería, gasfitería y construcción & Arte \\
Mecánica y transporte \\
Curanderismo \\
Comercio \\
Metalurgia y minería \\
Prensa \\
Religión \\
Textilería \\
Otros \\
\\
GRADO DE TRANSPARENCIA \\
Sufijación \\
Composición \\
Sintagmación \\
Transparente \\
Opaco \\
\end{tabular}

\section{Método}

\subsection{Tipo de investigación}

El presente trabajo es una investigación aplicada, descriptivo-comparada, no experimental, transeccional exploratoria y de corte documental. 


\subsection{Método de investigación}

El método empleado para esta investigación fue el análisis lexicográfico comparado de los oficios presentes en los trece diccionarios de peruanismos publicados a la fecha en soporte físico.

\subsection{Diseño de investigación}

El diseño específico de investigación es cuantitativo y cualitativo.

\subsection{Muestra/base de datos}

El corpus de vaciado del que se extrajeron los oficios estuvo compuesto por los diccionarios de peruanismos que citamos a continuación:

\section{Tabla 2}

Corpus de diccionarios

\begin{tabular}{lccc}
\hline N. $^{\circ}$ & DICCIONARIO & AUTOR & AÑO \\
\hline D1 & Diccionario de peruanismos & Juan de Arona & 1938 \\
D2 & Peruanismos & Martha Hildebrandt & 1969 \\
D3 & Argot limeño & Guillermo Bendezú & 1977 \\
D4 & Diccionario de peruanismos & Juan Álvarez Vita & 1990 \\
D5 & Vocabulario de peruanismos & Miguel Ugarte Chamorro & 1997 \\
D6 & El babla culta & Martha Hildebrandt & 2000 \\
D7 & Habla jugador & Julio Hevia & 2008 \\
D8 & Diccionario de peruanismos & Juan Álvarez Vita & 2009 \\
D9 & 1000 palabras y frases peruanas & Martha Hildebrandt & 2010 \\
D10 & Léxico popular peruano & Luisa Portilla & 2011 \\
D11 & Voces del español del Perú & Portilla y Ferrel & 2011 \\
D12 & Peruanismos & Martha Hildebrandt & 2013 \\
D13 & DiPerú & Academia Peruana de la Lengua & 2015 \\
\hline
\end{tabular}


Tanto la población como la muestra de estudio de esta investigación se corresponden, ambas lexicográficas antes enunciadas.

3.5. Instrumento de recolección de datos y técnicas de procesamiento de datos

Para los fines de este trabajo de investigación, la codificación y tabulación de los datos se llevó a cabo en hojas de cálculo Excel. Los datos tabulados en Excel se transfirieron al software SPSS para obtener las tablas de estadística descriptiva.

\section{Resultados y discusión}

\subsection{Oficios por frecuencia de aparición}

\section{Tabla 3}

Oficios por diccionario

\begin{tabular}{cc}
\hline DICCIONARIOS & $\begin{array}{c}\text { N. }{ }^{\circ} \text { DE } \\
\text { OFICIOS }\end{array}$ \\
\hline D1 & 20 \\
D2 & 14 \\
D3 & 8 \\
D4 & 150 \\
D5 & 119 \\
D6 & 6 \\
D7 & 4 \\
D8 & 158 \\
D9 & 157 \\
D10 & 3 \\
D11 & 8 \\
D12 & 20 \\
D13 & 238 \\
\hline
\end{tabular}

Tal como puede desprenderse de la tabla 3, el Diccionario de peruanismos (DiPerú) presenta el mayor número de oficios (238). Seguidamente, a 
considerable distancia, el Diccionario de peruanismos (2009), de Juan Álvarez Vita, y Mil palabras y frases peruanas, de Martha Hildebrandt, con 158 y 157 oficios, respectivamente.

A corta distancia de estas dos últimas obras se ubican las fuentes lexicográficas Vocabulario de peruanismos (1997), de Miguel Ugarte Chamorro, y la primera edición del Diccionario de peruanismos (1990), de Juan Álvarez Vita.

Tabla 4

Porcentaje de oficios por entradas de diccionarios

\begin{tabular}{ccc}
\hline DICCIONARIO & $\begin{array}{c}\text { \% DE OFICIOS } \\
\text { POR ENTRADAS } \\
\text { LÉXICAS }\end{array}$ & $\begin{array}{c}\text { NÚMERO TOTAL } \\
\text { DE ENTRADAS } \\
\text { LÉXICAS }\end{array}$ \\
\hline D1 & $1,0 \%$ & 1920 \\
D2 & $9,0 \%$ & 450 \\
D3 & $0,1 \%$ & 7220 \\
D4 & $2,0 \%$ & 7420 \\
D5 & $1,6 \%$ & 7300 \\
D6 & $0,2 \%$ & 3000 \\
D7 & $0,3 \%$ & 1650 \\
D8 & $2,0 \%$ & 17500 \\
D9 & $15,7 \%$ & 1000 \\
D10 & $0,08 \%$ & 368 \\
D11 & $0,9 \%$ & 888 \\
D12 & $8,0 \%$ & 250 \\
D13 & $2,0 \%$ & 10500 \\
\hline
\end{tabular}

Sin embargo, si cruzamos el número de oficios con el número de entradas de cada diccionario, será Mil palabras y frases peruanas de Martha Hildebrandt la fuente lexicográfica que ocupa el primer lugar con un $15,7 \%$. Es oportuno resaltar que la inclusión de oficios en los diccionarios de peruanismos siempre está supeditada al criterio del lexicógrafo que los incluye. Ello queda corroborado por el elevado 
número de ellos contenidos en la obra en la que participaron mayor número de lexicógrafos, caso de DiPerú. Otro argumento a favor de esta hipótesis es que la segunda obra lexicográfica de Juan Álvarez Vita añade únicamente ocho oficios a pesar de los 20 años de diferencia que existe entre ellas.

\section{Tabla 4a}

Recurrencia de oficios

\begin{tabular}{ccc}
\hline $\mathbf{N .}^{\circ}$ & OFICIOS & RECURRENCIA \\
\hline 1 & gasfitero & 8 \\
2 & suertero & 7 \\
3 & jalador & 6 \\
4 & caporal & 5 \\
5 & chanchero & 5 \\
6 & cuartelero & 5 \\
7 & emolientero & 5 \\
8 & huesero & 5 \\
9 & humitero & 5 \\
10 & lampero & 5 \\
11 & misturero & 5 \\
12 & panteonero & 5 \\
13 & tambero & 5 \\
14 & yerbero & 5 \\
\hline
\end{tabular}

Los resultados de la tabla muestran que ningún oficio aparece en los trece diccionarios del corpus de vaciado. El oficio con presencia en ocho de los trece diccionarios es gasfitero, préstamo adaptado del inglés gasfitter, definido por Martha Hildebrandt (2013) como el «obrero que instala y repara tuberías u otros artefactos del servicio de agua potable o alcantarillado», y a un punto porcentual de diferencia con suertero 'vendedor de loterías' y jalador, cuyo amplio campo semántico será abordado en la tabla 10 .

Los diez oficios restantes con mayor recurrencia en el corpus de estudio figuran en cinco diccionarios (38\%). Una particularidad de estos 
oficios es que han sido formados en el $85 \%$ de los casos mediante bases léxicas acompañadas por el sufijo nominal -ero en forma exclusivamente masculina, que dan cuenta, como afirma Muñoz, de oficios con menor grado de especialización (2010).

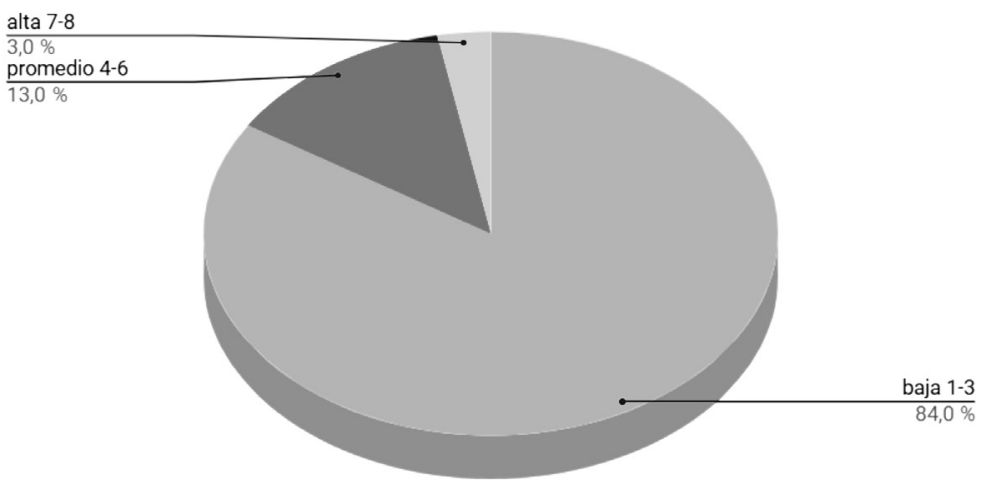

Gráfico 1. Recurrencia de oficios en diccionarios

El gráfico 1 muestra en forma categórica un elevadísimo $84 \%$ de oficios contenidos en los diccionarios de peruanismos con una baja frecuencia de aparición (figura en uno o dos diccionarios), un escaso 13 $\%$ con un promedio (aparece entre cuatro y seis diccionarios), e ínfimo $3 \%$ con una elevada (se consigna en siete u ocho diccionarios).

La baja recurrencia de oficios en el corpus de estudio puede relacionarse con el elevado porcentaje alcanzado por el campo de especialización OTROS en el gráfico 2.

A partir de estos resultados, podría deducirse que la restringida representatividad de los oficios peruanos es atribuible al sesgo inevitable de los lexicógrafos al seleccionar los oficios característicos del Perú, quienes basan su inclusión en factores, tales como la experiencia de vida, las afinidades, preferencias y no aspectos técnicos como la identificación de las ocupaciones en función de bases de datos como la elaborada por el INEI (2015). 


\subsection{Oficios peruanos por campos de especialidad}

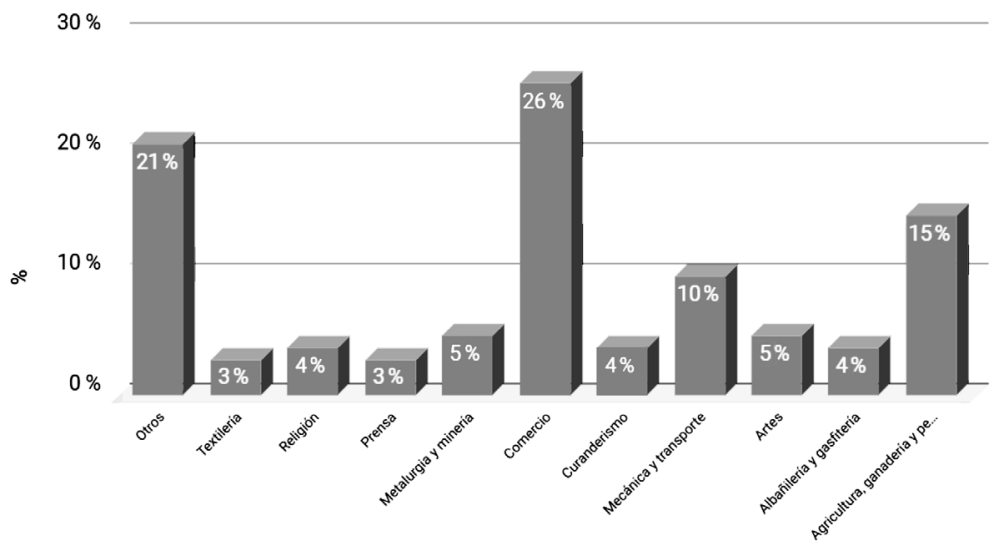

Gráfico 2. Oficios por campo de especialidad

Según el gráfico 2, el campo COMERCIO reunió la mayor cantidad de oficios (26\%). Las ocupaciones vinculadas con la tripleta ocupacional AGRICULTURA-GANADERÍA-PESCA, al igual que la dupleta MECÁNICA-TRANSPORTE obtuvieron el tercer y cuarto lugar en la concentración de oficios (15 y $10 \%$ respectivamente).

Es destacable señalar que el segundo lugar lo logró el ítem OTROS $(21 \%)$, que alberga oficios con muy baja recurrencia (1-2 \%) y de índole extremadamente heterogénea, cuya heterogeneidad, tal como manifiesta Muñoz (2010), corresponde a la amplia gama de ocupaciones a las que hacen referencia los sufijos relativos a profesiones, oficios u ocupaciones.

A nuestro entender, este considerable desbalance en los oficios muestra, a su vez, la escasa exhaustividad aplicada en las obras lexicográficas para explotar los campos semánticos ocupacionales peruanos. 
Dichos resultados, asimismo, demuestran un sesgo por parte de los lexicógrafos tanto en obras lexicográficas monoautorales como en biautorales y colectivas, que concentran su atención únicamente en tres de los diez grupos ocupaciones incluidos en la clasificación del INEI (2015), a saber, vendedores de comercios y mercados, trabajadores pesqueros, agropecuarios y de la mecánica.

Tabla 5

Algunos ejemplos de oficios peruanos por campo de especialidad

\begin{tabular}{|c|c|c|c|}
\hline COMERCIO & PESCA & AGRICULTURA & GANADERÍA \\
\hline rocotero & mitayero & viñatero & rodeador \\
\hline $\begin{array}{l}\text { Vendedor de } \\
\text { rocoto. } \\
\text { UCH }\end{array}$ & $\begin{array}{l}\text { Persona que se } \\
\text { dedica a la caza o } \\
\text { pesca. } \\
\text { JAV }\end{array}$ & $\begin{array}{l}\text { Persona que cultiva } \\
\text { viñas. } \\
\text { JAV }\end{array}$ & $\begin{array}{l}\text { Persona que } \\
\text { cuida el ganado } \\
\text { que quedará en } \\
\text { las chacras o será } \\
\text { vendido. } \\
\text { JAV }\end{array}$ \\
\hline ronero & peñero & desmontero & repuntero \\
\hline $\begin{array}{l}\text { Vendedor o } \\
\text { fabricante de } \\
\text { ron. } \\
\text { DP }\end{array}$ & $\begin{array}{l}\text { Pescador de } \\
\text { especies de las } \\
\text { peñas. } \\
\text { DP, JAV }\end{array}$ & $\begin{array}{l}\text { Agricultor que cultiva } \\
\text { cacao, arbustos o platas, } \\
\text { y recibe a cambio una } \\
\text { parte de la cosecha. } \\
\text { JAV }\end{array}$ & $\begin{array}{l}\text { Pastor que presta } \\
\text { sus servicios por } \\
\text { contrato. } \\
\text { JAV }\end{array}$ \\
\hline sanguchero & tarrafero & aviado & laceador \\
\hline $\begin{array}{l}\text { Vendedor de } \\
\text { sanguches. } \\
\text { DP }\end{array}$ & $\begin{array}{l}\text { Pescador } \\
\text { artesanal. } \\
\text { JAV }\end{array}$ & $\begin{array}{l}\text { Trabajador que recibe } \\
\text { mercancías al crédito } \\
\text { JAV }\end{array}$ & $\begin{array}{l}\text { Experto en el } \\
\text { manejo del lazo } \\
\text { para sujetar } \\
\text { animales } \\
\text { JAV }\end{array}$ \\
\hline
\end{tabular}

\section{UCH: DP de Ugarte Chamorro; DP: DiPerú; JAV: DP de Juan Alvarez Vita}

La tabla 5 evidencia que la mayor parte de estos oficios han sido formados a través de la adjunción del sufijo -ero a diversas bases léxicas 
que, en su mayor parte corresponden, como indica la NGLE (2009), a actividades de naturaleza menos profesional.

Entre los ejemplos presentados en esta misma tabla pueden identificarse oficios con marca dialectal pertenecientes a la Amazonía (aviado y mitayero) y a los Andes (repuntero).

\subsection{Oficios peruanos por recursos de formación}

\section{OFICIOS POR RECURSOS DE FORMACIÓN}

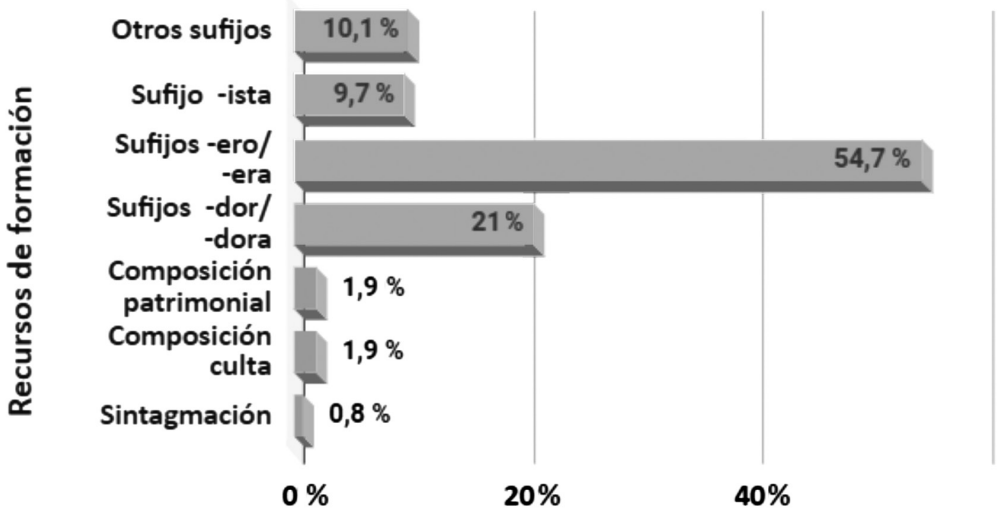

Gráfico 3. Recursos de formación

A partir de la lectura del gráfico 3, se deduce a todas luces que los sufijos comprenden el $95 \%$ de los recursos utilizados para la formación de peruanismos ocupacionales, y que el sufijo -erolera alcanza un elevado 54,7\% de productividad, seguido por -dor/-dora que obtiene un $21 \%$ e -ista, a mediana diferencia del anterior, con un 9,7\%. Es notoria, asimismo, la baja incidencia de los recursos de composición y sintagmación $(5 \%)$. 
Rosa LUNA

https://doi.org/10.46744/bapl.201902.001

Tabla 6

Ejemplos de sufijos más recurrentes en -ero/-era y -dor/-dora

\begin{tabular}{cc}
\hline SUFIJO -ero/-ra & SUFIJO -dor/-ra \\
\hline cajonero & amansador \\
aguatero & animador \\
avisero & auscultador \\
bicicletero & degustador \\
ayahuasquero & desenllantador \\
\hline
\end{tabular}

La tabla 6 incluye algunos ejemplos de los sufijos -ero y -dor, que tienen en común su relación con ocupaciones menos formales (Muñoz, 2010).

Tabla 7

Algunos ejemplos de oficios acabados en -ista

1. arreglista, sonidista, charanguista, tecladista, retablista, arpillerista

2. motorista, mototaxista, tractorista, trasladista

3. emergencista, dietista, tributaria, sectorista, serigrafista

Los ejemplos presentados confirman lo expuesto por Muñoz (2010), en cuanto a los campos de especialización prototípicos del sufijo -ista: música y arte (arreglista, sonidista, retablista, etc.), transporte (motorista, mototaxista, etc.), así como al mayor grado de especialización que lo caracteriza (emergencista, dietista, tributarista, etc.).

Tabla 8

Alternancia sufijal doble

\begin{tabular}{ccc}
\hline -adorl-ista & -erolador & -erol-ista \\
\hline charolador-charolista & buesero-sobador & tamalero-tamalista \\
rematador-rematista & cajonero-cajoneador & combero-combista \\
\hline
\end{tabular}


En esta tabla, puede observarse la alternancia en los pares -dor/ista, -ero/-dor y -ero/ista, que caracterizan a los sufijos nominales referidos a oficios (NGLE, 2009).

Tabla 9

Alternancia de base lexical con el mismo sufijo

\begin{tabular}{lll}
\hline gasfitero & plomero & fontanero \\
bodeguero & pulpero & \\
grifero & islero & \\
\hline
\end{tabular}

La tabla 9 ilustra ejemplos de alternancia de bases lexicales distintas que comparten el mismo sufijo y referente. De la primera alternancia triple, gasfitero, préstamo adaptado del inglés gasfitter, fue el más recurrente en el corpus lexicográfico (8 de los 9 diccionarios); mientras que las variantes denominativas plomero, también préstamo adaptado de inglés plumber, al igual que fontanero — que se utilizan más en en España y algunos países de Hispanoamérica - obtuvieron una baja recurrencia (3 de 13) (Hildebrandt, 2013).

La alternancia bodeguero/pulpero también difiere, en cuanto a su recurrencia en las fuentes de procedencia. Pulpero presenta una aparición bastante menor que bodeguero, que debido a su naturaleza arcaica figura únicamente en los diccionarios más antiguos.

Si bien la frecuencia de aparición del par denominativo griferol islero es bajísima (dos y una, respectivamente), vale precisar que islero, ra solo figura en el diccionario más reciente debido a su carácter neológico (DiPerú, 2015). Este vocablo ha empezado a utilizarse a partir del momento en el que las estaciones de gasolina empezaron a denominarse islas. 
https://doi.org/10.46744/bapl.201902.001

\section{Tabla 10}

Alternancia de base léxica y sufijal con significados distintos

jalador

enganchador

1. Brujo experto en asuntos amorosos (UCH).

2. Persona encargada de contratar obreros por el enganche (UCH).

datero (DP).

2. Persona que capta clientes en la vía pública (DP). (DP).

4. Persona que guía a pie a los caballos tomándolos por las riendas (DP)
1. Persona que capta pasajeros para un vehículo de transporte público

3. Persona que atrae pasajeros en terminales para pasar contrabando

1. Persona que se encarga de recoger y transmitir a la redacción de un medio de difusión los datos con los cuales se prepara una crónica (JAV).

2. Persona que proporciona información de importancia a terceros (DP, MH).

3. Hip. Persona que proporciona datos sobre caballos que ganarán en una carrera (UCH, DP).

4. Transp. Persona que brinda datos sobre el tiempo que media entre las diferentes unidades de transporte de una misma ruta para que aceleren o detengan el vehículo según su conveniencia (DP).

DP: DiPerú; UCH: Ugarte Chamorro; MH: Martha Hildebrandt; JAV: Juan Álvarez Vita

En la tabla 10, se incluyen tres oficios con más de un significado, dos de ellos polisémicos, caso de jalador y datero, y uno bisémico (enganchador). Las tres primeras acepciones de jalador están vinculadas con las personas encargadas de captar a otras, bien como pasajeros, clientes, contrabandistas, y la última de conducir no a personas, sino a animales.

En el caso de datero, el género próximo común es facilitar datos, y las diferencias específicas están referidas al campo en el que se emplean 
dichos datos: la redacción periodística, la investigación policial, la hípica y el transporte público. Finalmente, enganchador se aplica tanto al ámbito de la brujería como al de la construcción.

\subsection{Oficios peruanos por grado de transparencia}

\section{OFICIOS POR GRADO DE TRANSPARENCIA}

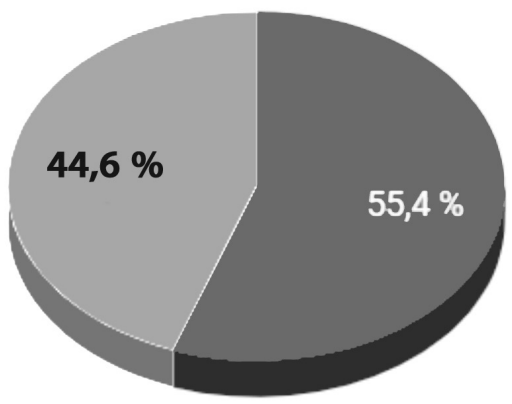

- Transparentes Opacos

Gráfico 4. Grado de transparencia

El gráfico 4 trasluce una relativa diferencia $(10 \%)$ porcentual entre los peruanismos de oficios transparentes o motivados y opacos o arbitrarios, según la terminología propuesta por Ullmann (1987).

\section{Tabla 11}

Ejemplos de oficios transparentes y opacos

\begin{tabular}{cc}
\hline TRANSPARENTES & OPACOS \\
\hline cebichero & cucharero \\
mototaxista, taxicholero & cocinero \\
\hline
\end{tabular}


La tabla 11 incluye ejemplos de sufijos adjuntos a bases léxicas transparentes y opacas. Estos últimos, en su totalidad, son falsos amigos intralingüísticos por cuanto su sentido no se deriva de la suma de los significados de sus bases léxicas y respectivos sufijos. En otras palabras, cocinero no alude a "quien cocina», sino a la "persona que hace las veces de laboratorista en la elaboración de cocaína» (JAV, 2009); cucharero no es el «vendedor de cucharas», sino el «médico o curandero que practica abortos» (JAV, 2009). En ambos casos, se trata de una relación semántica, que aun cuando metonímica del tipo instrumento-agente, resultan opacas por no estar convencionalizadas (Ullmann, 1987).

\section{Conclusiones y recomendaciones}

A continuación, presentamos las conclusiones a las que se ha arribado a partir de los datos analizados en el análisis y la discusión:

- El comportamiento lexicográfico de los oficios en los diccionarios de peruanismos tendencia a la derivación sufijal, semitransparencia, heterogeneidad temática y escasa recurrencia.

- El diccionario DiPerú es el más productivo en términos de presencia de oficios, seguido a gran distancia por el Diccionario de peruanismos, El habla castellana del Perú y Peruanismos, de Juan Álvarez Vita y Martha Hildebrandt, respectivamente.

- El índice de frecuencia de aparición de oficios en los diccionarios de peruanismos es bajo (entre 1 y 2 ).

- La sufijación es un recurso hiperproductivo en los oficios de los diccionarios de peruanismos. La mayor productividad sufijal la ostentan los sufijos -ero/-era y -ador/-dora. Los oficios suertero, jalador y gasfitero presentan el mayor índice de frecuencia léxica en los diccionarios de peruanismos.

- Los campos de especialización de oficios son muy heterogéneos, destacando de manera especial los oficios vinculados con el comercio.

- Los oficios presentan una semitransparencia léxico-semántica en el corpus de diccionarios de peruanismos. 


\section{BIBLIOGRAFÍA}

ALMELA, R. (1999). Procedimientos para la formación de palabras. Barcelona: Ariel.

CALVO, J. (Dir.) (2015). DiPerú. Diccionario de peruanismos. Lima: Academia Peruana de la Lengua.

ÁLVAREZ, J. (1990). Diccionario de peruanismos. Lima: Studium.

ÁlVAREZ, J. (2009). Diccionario de peruanismos. El habla castellana del Perú. Lima: Universidad Alas Peruanas.

BENDEZÚ, G. (1977). Argot limeño o jerga criolla del Perú. Lima: Lima S.A.

CABRÉ, T., FREIXA, J. y SOLÉ, E. (2000). Léxic i Neologia. Barcelona: IULA Univesitat Pompeu Fabra.

DOMÉNECH, M., ESTOPÁ, R. FOLIA, M., y MOREL, J. (2000). Neologismos de noms d'officis i de professions formats per sufixació en La neologia en el tombant de segle. Barcelona: IULA.

DE ARONA, J. (1983). Diccionario de peruanismos. Tomos I y II. Lima: Biblioteca Peruana.

DOMÍNGUEZ, V. (2016). Procesamiento de palabras en español: influencia de la frecuencia léxica y la transparencia semántica. Departamento de Psicología Cognitiva, Social y Organizacional Universidad de La Laguna

FUENTES, CAÑETE, HERDING, KOTZ Y PECCHI (2010). «Productividad del sufijo -ero en la neología del español de Argentina, Chile y Uruguay». Revista Signos, 43(72), 49-69 
https://doi.org/10.46744/bapl.201902.001

HEVIA, J. (2008). Habla jugador. Gajes y oficios de la jerga peruana. Lima: Taurus.

HILDEBRANDT, M. (1969). Peruanismos. Lima: Biblioteca Nacional del Perú.

. (2000). El habla culta (o lo que debiera serlo). Lima: Peisa. . (2010). 1000 palabras y frases peruanas. Lima: Espasa. . (2013). Peruanismos. Lima: Espasa.

INEI (2016). Clasificador Nacional de Ocupaciones 2015. Lima: Perú.

LUNA, R. (2010). «La cosificación como recurso terminológico de modalidades laborales en la economía peruana. Congreso de Investigaciones Lingüístico-filológicas». Lima.

MUÑOZ, L. (2010). La historia de los derivados -ismo -ista en el español moderno. (Tesis doctoral). Barcelona: Universidad Autónoma de Barcelona.

PORTILLA, L. (2011). Léxico popular peruano. Lima: Universidad Ricardo Palma.

PORTILLA, L. y FERREL, M. (2011). Voces del español del Perú. Lima: Universidad Ricardo Palma.

ROSSOWOVÁ, L. (2009). Sufijos nominales en español. Magisterská diplomová práce. Brno.

SKOLNIKOVÁ, A. (2008). «La productividad de los sufijos nominalizados deverbales en el español actual». Probebla, 11, 6-15

UGARTE, M. A. (1977). Vocabulario de peruanismos. Lima: Universidad Nacional Mayor de San Marcos. 
ULLMANN, S. (1987). Semántica. Introducción al conocimiento del significado. Madrid: Aguilar.

\section{ANEXO 1 \\ Listado de oficios peruanos en diccionarios de peruanismos (1938-2015)}

\begin{tabular}{|c|c|c|c|c|c|}
\hline abarrotador & capeador & cumananero & infografista & pailero & retacero \\
\hline abortero & capero & cumbiambero & inquisidor & pajareador & $\begin{array}{c}\text { rezador/ } \\
\text { rezandero }\end{array}$ \\
\hline adobero & capitulero & cutrero, ra & jalador, ra ${ }^{1}$ & pajarero & $\begin{array}{c}\text { rezador/ }^{2} \\
\text { rezandero }\end{array}$ \\
\hline aeromoza & caporal & damasquedor & jalador, $\mathrm{ra}^{2}$ & paletero & rocotero \\
\hline agenciero & carador $^{1}$ & dañero & jalador, ra ${ }^{3}$ & pallador, ra & rodeador \\
\hline $\begin{array}{l}\text { agente de } \\
\text { seguros }\end{array}$ & carador $^{2}$ & datero, $\mathrm{ra}^{1}$ & jardinera & pallaqueador & rompehuelgas \\
\hline $\begin{array}{l}\text { aguatero/ } \\
\text { tinajero, ra }\end{array}$ & caricatulista & datero, $\mathrm{ra}^{2}$ & jaspeador & pallaquero & rondero \\
\hline ahorrista & carbonero & decimista & jebero & $\begin{array}{l}\text { palmista/ } \\
\text { palmero }\end{array}$ & ronero, ra \\
\hline aimarista & careador & degustador & jornalero & $\begin{array}{c}\text { panteonero/ } \\
\text { sepultero }\end{array}$ & rosquetero \\
\hline ajicero -ra & $\begin{array}{l}\text { carguero/ } \\
\text { cargador }\end{array}$ & $\begin{array}{c}\text { desenfardelador, } \\
\text { ra }\end{array}$ & juguero, ra & papeletero & ruletero \\
\hline albero & carpero & desenllantador & jumbero & papero, ra & rumpólogo, ga \\
\hline almuercero, ra & carretillero, era & desmontero & jurero & paquetero, ra & sachero \\
\hline alpaquero, ra & carrilano & despachador & laborero & parador & sahumador, ra \\
\hline altarero & cateador & despenador & laceador & parquetero, ra & $\begin{array}{c}\text { salchipapero, } \\
\text { ra } \\
\end{array}$ \\
\hline alumbrante & catedrático & diagramador, ra & $\begin{array}{l}\text { lampero/ } \\
\text { lampeador }\end{array}$ & $\begin{array}{c}\text { paridor/ } \\
\text { partero }\end{array}$ & sanguchero, ra \\
\hline alzador, ra & $\begin{array}{l}\text { catipador/ } \\
\text { catipurero }\end{array}$ & dietista & laqueador & parrillero, ra & sectorista \\
\hline amansador & cauchero & digitador, ra & lauchero & pasquinista & semanero \\
\hline amarrador & chacador & diler & lavacarros & pasteador & senderólogo \\
\hline amazonista & chacarero & documentario & lechucero & pasador & sereno \\
\hline amauta & chalán & draguero & leñatero & pasteador & serigrafista \\
\hline ambulante & cebichero & enllantador, ra & levantador & patasquera & serumista \\
\hline anchovetero & chacarero & emergencista & lijador & patero & islero \\
\hline
\end{tabular}


Rosa LunA

https://doi.org/10.46744/bapl.201902.001

\begin{tabular}{|c|c|c|c|c|c|}
\hline andinista & chamán & emolientero, ra & limpialunas & patrullero & siringuero, ra \\
\hline animero & chambelán & enganchador $^{1}$ & limpionero, ra & pedalero, ra & sonidista \\
\hline animador & chamicero & enganchador $^{2}$ & llamadora & pedicurista & sorba \\
\hline anticuchero & $\begin{array}{c}\text { chancaquero, } \\
\text { ra }\end{array}$ & enganchado & llantero & pellejero & suertero, ra \\
\hline apoderado & $\begin{array}{c}\text { chancaquitero, } \\
\text { ra }\end{array}$ & enguayanchalor & llavero & peñero & tacachero, ra \\
\hline apostador & chanchero & enmaderador & lobista & perero & tacllero \\
\hline arenador & chacarero & escribano & lomero, ra & perforista & $\begin{array}{l}\text { tamalero/ } \\
\text { tamalista }\end{array}$ \\
\hline arguediano & chalador & espencero & loquero & $\begin{array}{l}\text { periodiquero/ } \\
\text { canillita }\end{array}$ & tambero \\
\hline armador & chamicero & estadígrafo & lundero & permisero & tamborero \\
\hline arpillerista & chanchero, ra & estandartero & lustrabotas & peruanista & tanganero \\
\hline arrancador & changador & esterillero & machero, ra & pescador & tarrajeador \\
\hline arreglista & chapeador & estibador & machetero & pesetero & tarrafero \\
\hline arrocero & chapero & estriptisero, ra & $\begin{array}{l}\text { machinero/ } \\
\text { machinguero }\end{array}$ & picantero, ra & tasquero \\
\hline asimilado & $\begin{array}{c}\text { charolador/ } \\
\text { charolista }\end{array}$ & expositor, ra & macumbero & picaronero, ra & taxicholero, ra \\
\hline auscultador & chancaquera & facilitador & madejero & piquero & tecladista \\
\hline autobusero & charanguista & farero & maderero & pinzador & tejador \\
\hline $\begin{array}{c}\text { ayahuasquero, } \\
\text { ra }\end{array}$ & chauchero & farmaceuta & maestro & pirata $^{1}$ & tejedora \\
\hline aviado & chaufero, ra & ferretero, ra & maleficiero & pirata $^{2}$ & telarista \\
\hline aviador & chavetero & feudatario & malero, ra & piritero & telefonista \\
\hline avionero & chavinero, ra & fidelero, ra & manguero & pishinero & $\begin{array}{l}\text { teniente } \\
\text { alcalde }\end{array}$ \\
\hline avisero & chicharronero & fierrero, ra & $\begin{array}{c}\text { manicero/ } \\
\text { manisero }\end{array}$ & pisquero, ra & teñidor \\
\hline azafata & chichero ${ }^{1}$ & fileteador & $\begin{array}{c}\text { manicurista/ } \\
\text { manicuro }\end{array}$ & pistero, ra & terramoza \\
\hline balancinero & chichero $^{2}$ & fletero & manquecero & planchador & textil \\
\hline bancario & chiclero, ra & financista & maquinista & planchero, ra & textilero, ra \\
\hline balsero & chifero, ra & fletero & marcador & popero & tinterillo \\
\hline barqueador & chiflera & foliculario & mariateguista & poronguero $^{1}$ & tipeador, ra \\
\hline baqueteador & chimbador & folletinero & maromero & poronguero $^{2}$ & titulero \\
\hline baquiano & chinchillero & fonomímico & maruchero, ra & portapliegos & tolvero \\
\hline barchilón & chinchorrero & fotocopista & maromero, ra & positario & tomero, ra \\
\hline basurero & chinganero & fotograbador & martillero & portuario & topiquero, ra \\
\hline
\end{tabular}


https://doi.org/10.46744/bapl.201902.001

\begin{tabular}{|c|c|c|c|c|c|}
\hline bicicletero & chirimoyero & fresquero & matero & prensero & tramitador, ra \\
\hline $\begin{array}{c}\text { bodeguero/ } \\
\text { pulpero }\end{array}$ & chirisuyero & $\begin{array}{l}\text { funcionario } \\
\text { público/ } \\
\text { funcionario }\end{array}$ & matizador & pretinero & tornicero \\
\hline boletero & chivatero & fundador & mayoliquero & procurador & torrejero \\
\hline bolichero & chivero & galvanizador & mayordomo & $\begin{array}{c}\text { proero/ } \\
\text { proel }\end{array}$ & tractorista \\
\hline bombardeador & choclero & gamonal & mazamorrero & pruebista & trasladista \\
\hline bonitero & choncholisero & ganchero & médico legista & $\begin{array}{c}\text { pusangueador/ } \\
\text { pusanguero }\end{array}$ & trencero \\
\hline brevetero, ra & chupetero & garcilacista & mejorero, ra & pututero & tributarista \\
\hline buñuelero & chunero & garitero & menudenciero & quepisero & triciclero \\
\hline busconero & cirujano & $\begin{array}{c}\text { gasfitero/ } \\
\text { fontanero/ } \\
\text { plomero }\end{array}$ & mesero & quimbalero, ra & trochero, ra \\
\hline butifarrero & cobrador & gaseosero, ra & microbusero, ra & $\begin{array}{c}\text { quiminquero, } \\
\mathrm{ra}\end{array}$ & tubero, ra \\
\hline cabecero, ra & cocalero, ra & gatero & $\begin{array}{l}\text { misturero/ } \\
\text { mixturero, ra }\end{array}$ & $\begin{array}{c}\text { quimbaletero, } \\
\text { ra }\end{array}$ & tunelero \\
\hline cabecista & cocinero & gobernador & mitayero, ra & rabona & urraco, ca \\
\hline cacaotero & coctelero & golpeteador & mortalero, ra & ranchero & tunero \\
\hline cachascanista & colectivero & golondrina & motero & ranero & utilero \\
\hline cafetero, ra & $\begin{array}{l}\text { combista/ } \\
\text { combicero/ } \\
\text { combero }\end{array}$ & gondolero, ra & motinista & raspadillero, ra & vallejista \\
\hline cajero, ra & compactado & grafotécnico & motocarrista & rastrero, ra & vareador \\
\hline $\begin{array}{l}\text { cajonero/ } \\
\text { cajoneador }\end{array}$ & $\begin{array}{l}\text { componedor, } \\
\text { dora }\end{array}$ & grageador & motorista & rayador $^{1}$ & vargasllosista \\
\hline cajuelero & composturero & $\begin{array}{l}\text { grifero, ra } \\
\text { /islero, ra } \\
\end{array}$ & motorizado & rayador $^{2}$ & vegetalista \\
\hline calichero, ra & concertero & guardatiempo & mototaxista & rebajador & vicuñero \\
\hline calpeador & conchero, ra & guayanchero & moyador & recalador & vinero, ra \\
\hline camarero & conductor & hacedora & muñidor & recaudador & viñatero, ra \\
\hline camaretero & conferencista & hacendado & natacha & recaudera & vivandero, ra \\
\hline camaronero, ra & contralor & helador & nievero & $\begin{array}{c}\text { recibidora// } \\
\text { rebuscadora/ } \\
\text { arrastradora, } \\
\text { lavandera, } \\
\text { marquesina/ } \\
\text { patinadora/ } \\
\text { meretriz/ } \\
\text { visitadora/ } \\
\text { chivatera }\end{array}$ & vocal \\
\hline
\end{tabular}


RosA LUNA

https://doi.org/10.46744/bapl.201902.001

\begin{tabular}{|c|c|c|c|c|c|}
\hline $\begin{array}{c}\text { cambalachero/ } \\
\text { trapalón/ } \\
\text { camorrista } \\
\end{array}$ & contraplacador & $\begin{array}{l}\text { huachimán/ } \\
\text { guachimán }\end{array}$ & noventero & reciclador, ra & viñatero \\
\hline cambista & copetinera & $\begin{array}{c}\text { huaquero/ } \\
\text { guaquero }\end{array}$ & ñaupador & recursero & vivandera \\
\hline camionero & coquero, ra & huarayero, ra & obrero & redimidor & volantero, ra \\
\hline campanero & cornetero -ra & huaripolera & obstetriz & regente & $\begin{array}{c}\text { yerbatero/ } \\
\text { yerbero/ } \\
\text { hierbatero, ra }\end{array}$ \\
\hline canastero, ra & coreuta & huaycholero & oidor & regidor, ra & yuquero, ra \\
\hline canchador $^{1}$ & cosechador & huayrero & ojotero, ra & rehabilitador & yutero \\
\hline canchador $^{2}$ & cosmiatra & huertero & omnibusero & $\begin{array}{l}\text { rematista/ } \\
\text { rematador }\end{array}$ & yuyero \\
\hline canchaminero & criandero & $\begin{array}{l}\text { huesero/ } \\
\text { sobador }\end{array}$ & orero & repelador & zarandeador \\
\hline canchero ${ }^{1}$ & criero & humitero & pabilero, ra & repiqueteador & \\
\hline canchero $^{2}$ & crucerista & imprentero & pacayero & repuntero & \\
\hline cangallero & cuartelero, ra & impulsador & $\begin{array}{c}\text { pachamancólogo/ } \\
\text { pachamanquero }\end{array}$ & rescatista & \\
\hline cantor & cubicador & incaiquero & pacotillero & reponsero & \\
\hline cañavelero & cucharero & infectólogo & $\begin{array}{l}\text { paichero/ } \\
\text { paichetero }\end{array}$ & retablista & \\
\hline
\end{tabular}

